\title{
PDMS-Au/Ag Nanocomposite Films as Highly Sensitive SERS Substrates ${ }^{\dagger}$
}

\author{
Attila Bonyár 1,* , Zsanett Izsold ${ }^{1}$, Alexandra Borók ${ }^{1}$, István Csarnovics ${ }^{2}$, László Himics ${ }^{3}$, \\ Miklós Veres ${ }^{3}$ and Gábor Harsányi ${ }^{1}$ \\ 1 Department of Electronics Technology, Budapest University of Technology and Economics, \\ H-1111 Budapest, Hungary; izsoldzsanett@gmail.com (Z.I.); borokalek@gmail.com (A.B.); \\ harsanyi@ett.bme.hu (G.H.) \\ 2 Department of Experimental Physics, University of Debrecen, H-4032 Debrecen, Hungary; \\ csarnovics.istvan@science.unideb.hu \\ 3 Institute for Solid State Physics and Optics, Wigner Research Centre for Physics of the Hungarian \\ Academy of Sciences, H-1121 Budapest, Hungary; himics.laszlo@wigner.mta.hu (L.H.); \\ veres.miklos@wigner.mta.hu (M.V.) \\ * Correspondence: bonyar@ett.bme.hu; Tel.: +36-1-463-2758 \\ + Presented at the Eurosensors 2018 Conference, Graz, Austria, 9-12 September 2018. \\ Published: 26 November 2018
}

\begin{abstract}
Polydimethylsiloxane (PDMS)-gold/silver nanoparticle composite films were synthetized in situ by using a simple method, which is based on the reduction of chloroauric acid $\left(\mathrm{HAuCl}_{4}\right)$ or silver nitrate solution $\left(\mathrm{AgNO}_{3}\right)$ by the PDMS membrane. A method to monitor the development of the plasmonic absorbance peak in situ (during the synthesis of the nanoparticles) is introduced in order to provide a convenient way to investigate the effect of the technological parameters on the position of the resulting peak. The resulting composite films were tested as SERS substrates at two excitation wavelengths $(488 \mathrm{~nm}, 785 \mathrm{~nm})$ and the preliminary results indicate that they are promising candidates for this application, with high sensitivities accompanied by cheap fabrication costs.
\end{abstract}

Keywords: Raman-spectroscopy; SERS; nanocomposites; PDMS; gold; silver

\section{Introduction}

Surface enhanced Raman spectroscopy (SERS) is an analytical method which can significantly increase the yield of Raman scattering of target molecules adsorbed on the surface of metallic nanoparticles (the enhancement factor can even reach the order of $10^{10}-10^{11}$ ) [1]. However, depending on the plasmonic and geometrical properties of the nanostructures (particle size, shape, interparticle distance) and the operation wavelength, this enhancement can vary and optimization of the nanostructures are needed for outstanding enhancement. Hereby we investigate a straightforward approach for the fabrication of PDMS-Au/Ag nanocomposite films for this purpose. In this method, the silicon hydride (Si-H) groups of the residual curing agent in the PDMS elastomer act as reducing agents for direct nanoparticle synthesis on a PDMS membrane [2]. In this work, on the one hand, we aim to investigate the effect of synthesis parameters on this process in detail. For this purpose, a method is introduced which enables the direct real-time monitoring of the plasmonic peak during nanoparticle development in a microfluidic cell (Figure 1), with optical spectroscopy. In one of our previous works we demonstrated, that by increasing the temperature during nanoparticle synthesis, the incubation time needed to produce useable nanocomposites can be reduced from days to a couple of hours [3]. Our other major aim is to test these PDMS-Au/Ag nanocomposite films prepared at increased incubation temperatures as SERSsubstrates. 


\section{Materials and Methods}

\subsection{PDMS-Au/Ag Nanocomposite Preparation}

The PDMS samples were prepared by mixing SYLGARD ${ }^{\circledR} 184$ silicone elastomer with its corresponding curing agent in 1:5 mass ratio. The mixture was degassed in a vacuum exsiccator then poured to either a 3D printed mold to create microfluidic cells (Figure 1) or into a simple glass mold to form a $10 \mathrm{~cm} \times 10 \mathrm{~cm} \times 5 \mathrm{~mm}$ film, which was later cut to an approx. $2 \mathrm{~cm} \times 2 \mathrm{~cm}$ pieces for the SERS experiments. To increase the speed of polymerization the molding form was placed to a ceramic oven for $45 \mathrm{~min}$ at $80^{\circ} \mathrm{C}$. The microfluidic cell was finalized by bonding the PDMS part to a corona discharge treated glass slide. For the preparation of PDMS-Au/Ag nanoparticle composite films 100 $\mu \mathrm{L}$ of $2 \%(\mathrm{~m} / \mathrm{m})$ chloroauric acid $\left(\mathrm{HAuCl}_{4}\right.$, from Sigma Aldrich (Saint Louis, MO, USA)) or $2 \%(\mathrm{~m} / \mathrm{m}$ ) silver nitrate solution $\left(\mathrm{AgNO}_{3}\right.$, also from Sigma Aldrich) was pipetted on top of the PDMS blocks, or injected into the microfluidic cell, both shown in Figure 1. The microfluidic cells were hermetically sealed during incubation, also, the PDMS block samples were put into a hermetically sealed Petri dish, along with drops of water, to avoid the evaporation of the precursor solution during incubation at higher temperatures. After the given incubation time the samples were rinsed with deionized water, dried then were subjected to optical spectroscopyimmediately.

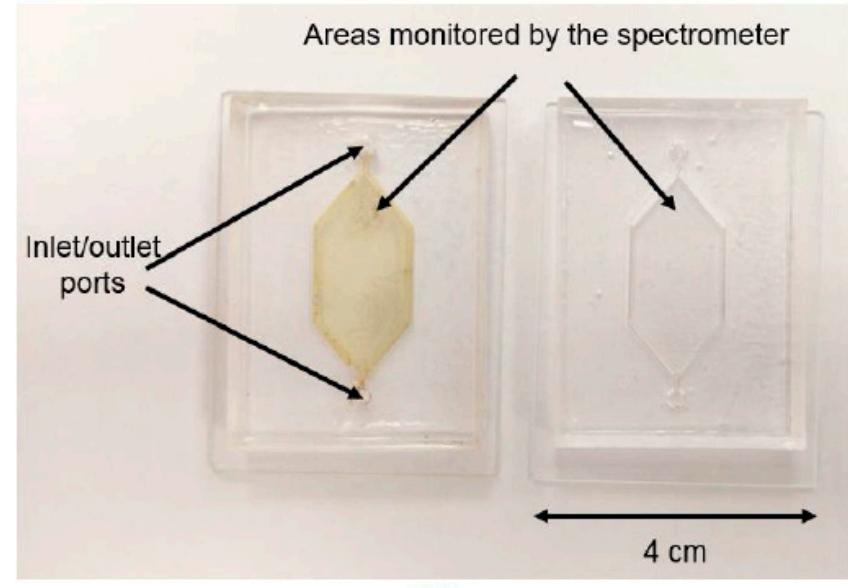

(a)

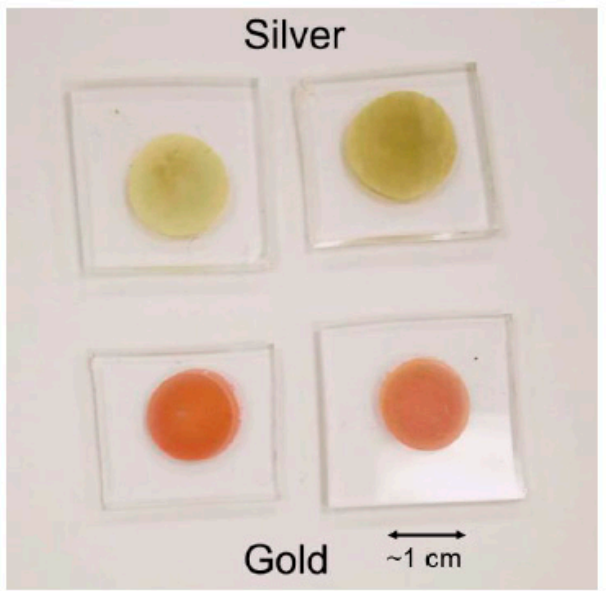

(b)

Figure 1. (a) The custom-designed PDMS microfluidic cell, which was used for the in situ monitoring of nanoparticle synthesis with optical spectroscopy Left: after a 2 days long silver nanoparticle synthesis; right: a freshly prepared cell. (b) PDMS-Ag (top row) and PDMS-Au (bottom row) nanocomposites prepared for the SERS experiments.

\subsection{Optical Spectroscopy}

For the real time monitoring of nanocomposite development and to measure the plasmon resonance spectra of the samples optical spectroscopy were performed with an Avantes Avaspec 2048-4DT spectrometer and an Avantes Avalight DHS halogen light source between $350 \mathrm{~nm}$ and 750 $\mathrm{nm}$. For the evaluation of the obtained spectra, a custom-written Matlab program was used.

\subsection{SERS Measurements}

SERS measurements were performed with a Renishaw 1000 micro-Raman spectrometer with two excitation wavelengths at $488 \mathrm{~nm}$ and $785 \mathrm{~nm}$. The diameter of the excitation spot was around $1 \mu \mathrm{m}$, which was monitored with a 50× objective. The spectra were recorded with $10 \mathrm{~s}$ integration time. The SERS enhancement of the composite samples was tested using a $1 \mathrm{mM}$ benzophenon-isopropyl alcohol solution by dripping the same volume onto the samples so that it formed a thin layer on the surface. As a reference, the same amount of test solution was dripped and measured on a clean $\mathrm{Si}$ wafer. The two characteristic peaks at $1590 \mathrm{~cm}^{-1}$ and $1660 \mathrm{~cm}^{-1}$ were used for the evaluation of sample performance. 


\section{Results and discussion}

\subsection{Real-Time Investigation of Nanocomposite Development}

The 3D plot of Figure 2 shows the development of the plasmonic peak of a PDMS-Ag nanocomposite film, monitored for 2 days in the microfluidic cell presented in Figure 1 with optical spectroscopy. The microfluidic cell provides great means to monitor the peak development at different incubation conditions, which enables the optimization of the synthesis parameters and the fine-tuning of the peak for SERS and/or LSPR (localized surface plasmon resonance) applications.

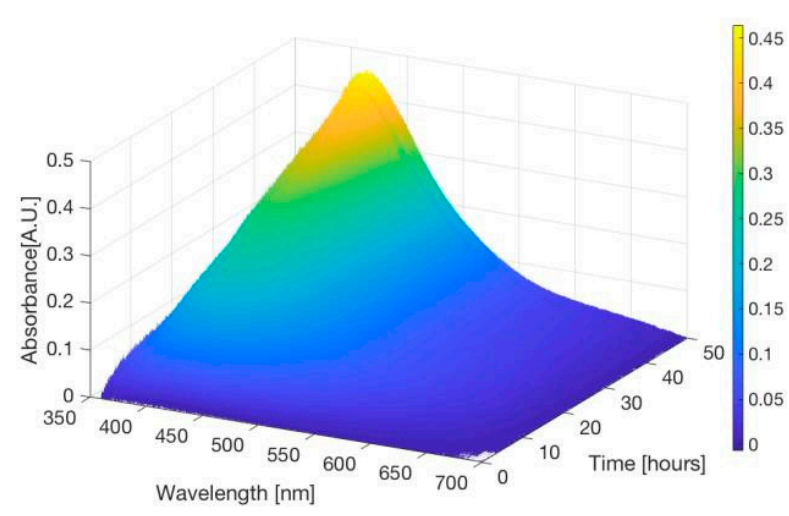

(a)

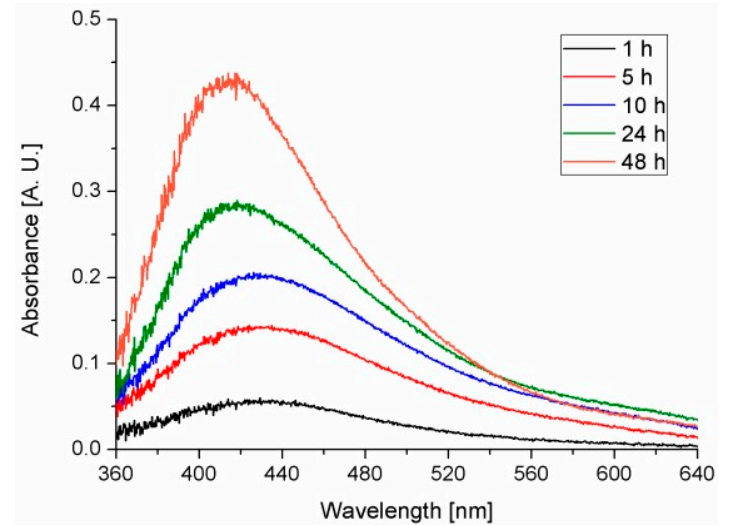

(b)

Figure 2. (a) $3 \mathrm{D}$ plot of the development of the plasmonic absorbance peak of a PDMS-Ag nanocomposite film. (b) Absorbance spectra of the same composite film after given time periods. A baseline drift correction was applied at the data, based on the drift measured at $700 \mathrm{~nm}$.

\subsection{SERS Results}

Figures 3 and 4 present sample SERS results, measured on PDMS-Au/Ag samples, which were prepared at higher incubation temperatures. The corresponding plasmonic absorbance spectra of two such samples can be seen in Figure 3a. The nanocomposite samples yielded substantial SERS signals at both excitation wavelengths $(488 \mathrm{~nm}$ and $785 \mathrm{~nm}$ ). Compared to the reference Raman signal

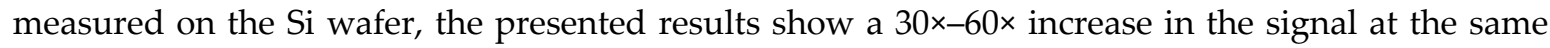
experimental conditions (thin fluid film of the test solution over the surface of the samples).

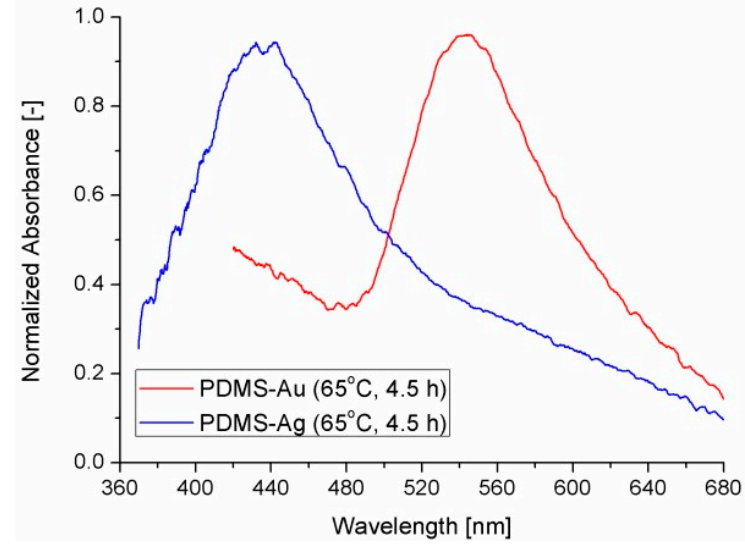

(a)

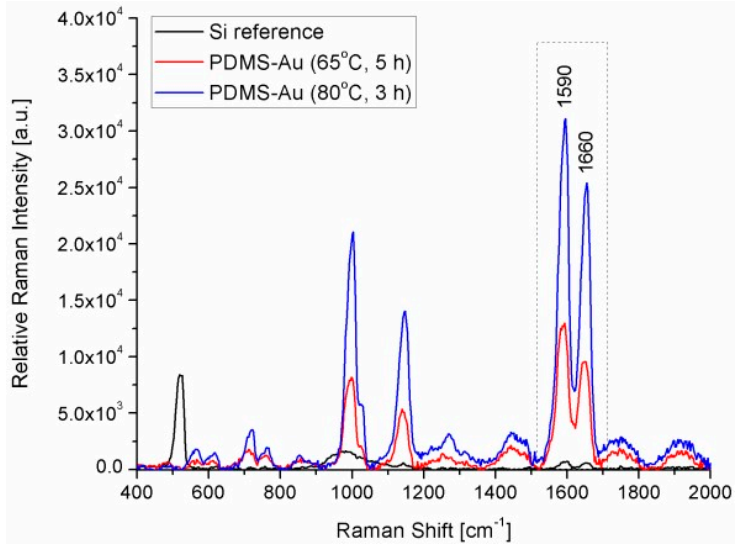

(b)

Figure 3. (a) Normalized absorbance spectra of two PDMS-Ag/Au samples prepared at an increased incubation temperature of $65^{\circ} \mathrm{C}$ for the SERS experiments. (b) Raman (Si reference) and SERS (PDMSAu nanocomposites) spectra of the of the benzophenon-isopropyl alcohol test solution measured with $488 \mathrm{~nm}$ excitation. 


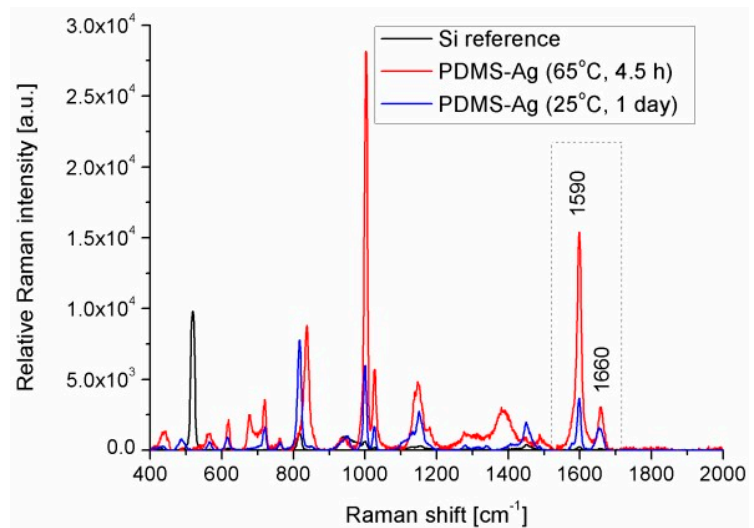

(a)

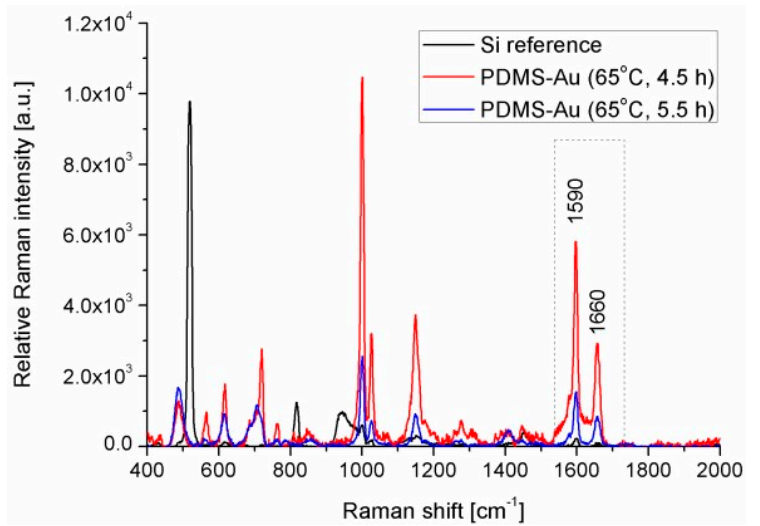

(b)

Figure 4. (a) Raman (Si reference) and SERS (PDMS-Ag nanocomposites) spectra of the of the benzophenon-isopropyl alcohol test solution measured with $785 \mathrm{~nm}$ excitation. (b) Raman (Si reference) and SERS (PDMS-Au nanocomposites) spectra of the of the benzophenon-isopropyl alcohol test solution measured with 785 nm excitation.

\section{Conclusions}

Our presented results demonstrate that the PDMS-Au/Ag nanocomposites can be viable candidates for highly sensitive and cheap SERS substrates. The effect of the synthesis parameters on the SERS enhancement, results obtained at $514 \mathrm{~nm}$ excitation and performance comparison with commercially available SERS substrates (at fixed experimental conditions) will be presented in the full paper.

Author Contributions: A.B. (Attila Bonyár) conceived, designed and supervised the experiments and wrote the paper. Z.I. and A.B. (Alexandra Borók) prepared the PDMS nanocomposites and performed the optical spectroscopy measurements. I.C. helped with the design of the experiments and the evaluation of the data. L.H. and M.V. performed the SERS experiments and evaluated the data. G.H. supervised the project.

Acknowledgments: The research reported in this paper was partially supported by the Higher Education Excellence Program of the Ministry of Human Capacities in the frame of Nanotechnology and Materials Science research and also Biotechnology research areas of Budapest University of Technology and Economics (BME FIKP-NAT and BME-FIKP-BIO). The research has also been partially supported by the "ÚNKP" program of the Hungarian Government. Attila Bonyár and Miklós Veres is grateful for the support of the János Bolyai Research Scholarship of the Hungarian Academy of Sciences.

Conflicts of Interest: The authors declare no conflict of interest. The founding sponsors had no role in the design of the study; in the collection, analyses, or interpretation of data; in the writing of the manuscript, and in the decision to publish the results.

\section{References}

1. Pamela, A. Mosier-Boss, Review of SERS substrates for chemical sensing. Nanomaterials 2017, 7,142.

2. SadAbadi, H.; Badilescu, S.; Packirisamy, M.; Wührich, R. Integration of gold nanoparticles in PDMS microfluidics for lab-on-a-chip plasmonic biosensing of growth hormones. Biosens. Bioelectron. 2013, 44, 77-84.

3. Bonyar, A.; Izsold, Z.; Himics, L.; Veres, M.; Csarnovics, I. Investigation of PDMS-gold nanoparticle composite films for plasmonic sensors. In Proceedings of the 23rd IEEE International Symposium for Design and Technology in Electronic Packaging (IEEE-SIITME), Constanta, Romania, 26-29 October 2017; pp. 25-28.

(C) 2018 by the authors. Licensee MDPI, Basel, Switzerland. This article is an open access article distributed under the terms and conditions of the Creative Commons Attribution (CC BY) license (http://creativecommons.org/licenses/by/4.0/). 